Ambiente \& Água - An Interdisciplinary Journal of Applied Science
ISSN 1980-993X - doi:10.4136/1980-993X
www.ambi-agua.net
E-mail: ambi.agua@gmail.com

\title{
Reviewers of Volume 12, 2017 of Revista Ambiente \& Água
}

\section{Masthead Section}

\author{
doi:10.4136/ambi-agua.1930 \\ Getulio Teixeira Batista \\ Editor of Revista Ambiente \& Água \\ e-mail: ambi.agua@gmail.com
}

The following list (Table 1) includes the names and affiliations of all reviewers that evaluated submissions processed for Volume 12, 2017 of Revista Ambiente \& Água. Including manuscripts that were accepted and published in one of the six annual issues and also manuscripts submitted and rejected up to October 10, 2017. It may therefore include the names of reviewers that completed their evaluations between September, 2016 and October, 2017.

We are deeply indebted to all of them that voluntarily and anonymously spent their precious time to support the journal, and contributed immensely to the quality of the published articles. We gratefully acknowledge their professional work and outstanding product.

Table 1. Ad Hoc Peer Reviewers that evaluated submissions for the 2017 editions of Revista Ambiente \& Água, Volume 12, n. 1, 2, 3, 4, 5, and 6, 2017.

Abd Elnaby Kabeel

Abelardo Antônio de Assunção Montenegro

Adeildo Cabral da Silva

Ademir Kleber Morbeck Oliveira

Ademir Paceli Barbassa

Adilson Ben da Costa

Adriana Maria Lotito

Adriana Patricia Gallego

Adriana Schueler

Adriana Sturion Lorenzi
Tanta University, Tanta, Gharbia, Egito

Universidade Federal Rural de Pernambuco (UFRPE), Recife, PE, Brasil

Instituto Federal de Educação, Ciência e Tecnologia do Ceará (IFCE), Fortaleza, CE, Brasil

Universidade Anhanguera (UNIDERP), Campo Grande, MS, Brasil

Universidade Federal de São Carlos (UFSCar), São Carlos, SP, Brasil

Universidade de Santa Cruz do Sul (UNISC), Santa Cruz do Sul, RS, Brasil

Apulian Regional Agency for Environmental Protection (ARPA - Puglia), Itália

Universidad Piloto de Colombia (UPC), Bogotá, Colômbia

Universidade Federal Rural do Rio de Janeiro (UFRRJ), Niterói, RJ, Brasil

Universidade de São Paulo - Escola Superior de Agricultura Luiz de Queiroz (ESALQ/USP), Piracicaba, SP, Brasil 
Adriane Martins de Freitas

Adriano Valentim Diotto

Adriano Weidner Cacciatori Marenzi

Adryane Gorayeb

Afonso de Liguori Oliveira

Alba Arana

Alberto Soares de Melo

Alcemar Rodrigues Martello

Alessandra Cristina Corsi

Alessandra Cristina Silva Valentim

Alexandre Dal Pai

Alexandre de Oliveira Lima

Alexandre Lioi Nascentes

Alexsandro Oliveira da Silva

Alfredo Pereira

Aline Falck

Aline Maria Meiguins de Lima

Álvaro José Back

Aminul Islam

Ana Aparecida da Silva Almeida

Ana Cristina Mielli

Ana Ghislane Henriques Pereira Van Elk

Ana Lucia Fonseca

Ana Paula Peron

Ana Paula Silva Camelo

Anderson Cristian Bergamin

Anderson Nascimento do Vasco
Universidade Tecnológica Federal do Paraná (UTFPR), Curitiba, PR, Brasil

Universidade Federal de Lavras (UFLA), Lavras, MG, Brasil

Universidade do Vale do Itajaí, (UNIVALI), Itajaí, SC, Brasil

Universidade Federal do Ceará (UFC), Fortaleza, CE, Brasil

Universidade Federal de Minas Gerais (UFMG), Belo Horizonte, MG, Brasil

Universidade do Oeste Paulista (Unoeste), Presidente Prudente, SP, Brasil

Universidade Estadual da Paraíba (UEPB), Campina Grande, $\mathrm{PB}$, Brasil

Universidade Estadual do Paraná (UNESPAR), União da Vitória, PR, Brasil

Instituto de Pesquisas Tecnológicas do Estado de São Paulo (IPT), São Paulo, SP, Brasil

Universidade Federal do Recôncavo da Bahia (UFRB), Cruz das Almas, BA, Brasil

Universidade Estadual Paulista Julio de Mesquita Filho (UNESP), Botucatu, SP, Brasil

Universidade do Estado do Rio Grande do Norte (UERN), Natal, RN, Brasil

Universidade Federal Rural do Rio de Janeiro (UFRRJ), Rio de Janeiro, RJ, Brasil

Universidade Federal do Ceará (UFC), Fortaleza, CE, Brasil

Ministério da Ciência Tecnologia e Inovação (MCTIC), Brasília, DF, Brasil

George Mason University, Fairfax, Virginia, Estados Unidos

Universidade Federal do Para (UFPA), Belém, PA, Brasil

Empresa de Pesquisa Agropecuária e Extensão Rural de Santa Catarina (EPAGRI), Florianópolis, SC, Brasil

Bangladesh Rice Research Institute, Gazipur, Bangladesh

Universidade de Taubaté (UNITAU), Taubaté, SP, Brasil

Universidade de São Paulo (USP), São Paulo, SP, Brasil

Universidade do Estado do Rio de Janeiro (UERJ), Rio de Janeiro, RJ, Brasil

Universidade Federal de Itajuba (UNIFEI), Itajubá, MG, Brasil

Universidade Federal do Piauí (UFPI), Picos, PI, Brasil

Instituto Federal de Educação, Ciência e Tecnologia de Brasília (IFB), Brasília, DF, Brasil

Universidade Federal de Rondônia (UNIR), Porto Velho, RO, Brasil

Instituto Federal de Sergipe (IFS), São Cristóvão, SE, Brasil 
André Luís de Gasper

André Quintão de Almeida

Andrea Almeida Cavalcante

Andrea Giuseppe Capodaglio

Andrea Nunes Vaz Pedroso

Andréa Rodrigues Marques

Andrea Salgado

Andreia Maria Anunciação Gomes

Andrew Dean

Angela Dulce Cavenaghi Altemio

Annibal Duarte Pereira Neto

Antonia Leila Rocha Neves

Antonio Domingues Benetti

Antonio Eduardo Leão Lanna

Antonio Laverde Junior

Antonio Sergio Ferreira Mendonça

Antonio Teixeira Matos

Aonghus McNabola

Argemiro Midonês Bastos

Ariovaldo Antonio Lucas

Athanasos Argyriou

Áurea Luiza Lemes da Silva

Bianca Ramos Pezzini

Bruno Mantovanelli

Caio Fernando Gromboni
Universidade Regional de Blumenau (FURB), Blumenau, SC, Brasil

Universidade Federal de Sergipe (UFS), São Cristóvão, Sergipe, Brasil

Universidade Estadual do Ceará (UECE), Fortaleza, CE, Brasil

Università Degli Studi di Pavia, Pavia, Itália

Instituto de Botânica (IBT), São Paulo, SP, Brasil

Centro Federal de Educação Tecnológica de Minas Gerais (CEFET-MG), Belo Horizonte, MG, Brasil

Universidade Federal do Rio de Janeiro (UFRJ), Rio de Janeiro, RJ, Brasil

Universidade Federal de Itajubá (UNIFEI), Itajubá, MG, Brasil

Manchester Metropolitan University, Manchester, Reino Unido

Universidade Federal da Grande Dourados (UFGD), Dourados, MS, Brasil

Universidade Federal Fluminense (UFF), Niterói, RJ, Brasil

Universidade Federal do Ceará (UFC), Fortaleza, CE, Brasil

Universidade Federal do Rio Grande do Sul (UFRGS), Porto Alegre, RS, Brasil

AlfaSigma Consultoria Sociedade Simples Ltda, Porto Alegre, RS, Brasil

Universidade Tecnológica Federal do Paraná (UTFPR), Londrina, PR, Brasil

Universidade Federal do Espírito Santo (UFES), Vila Velha, ES, Brasil

Universidade Federal de Minas Gerais (UFMG), Belo Horizonte, MG, Brasil

University of Dublin Trinity College, Dublin, Irlanda

Instituto Federal do Amapá (IFAP), Macapá, AP, Brasil

Universidade Federal de Sergipe (UFSE), São Cristóvão, SE, Brasil

School of Earth and Environmental Sciences (SEES), Portsmouth, Reino Unido

Universidade Federal de Santa Catarina (UFSC), Florianópolis, SC, Brasil

Universidade Federal de Santa Catarina (UFSC), Florianópolis, SC, Brasil

Universidade Federal Rural de Pernambuco (UFRPE), Recife, PE, Brasil

Instituto Federal de Educação, Ciência e Tecnologia da Bahia (IFBA), Ilhéus, BA, Brasil 
Camila Gonçalves Athanásio

Camille Ferreira Mannarino

Carlo Collivignarelli

Carlos Alexandre Borges Garcia

Carlos Grohmann

Carlos Henrique Gomes Martins

Carlos Montenegro

Carolina Lourencetti

Cassia Farias

Catarina da Silva Pedrozo

Celso Bandeira de Melo Ribeiro

Celso Souza Catelani

César Carmona-Moreno

Christiano Magini

Claudemir Marcos Radetski

Claudia Ribeiro

Claudio Fernando Mahler

Cláudio Leite de Souza

Claudivan Feitosa Lacerda

Cleber Ibraim Salimon

Cleber Souza Correa

Cleder Somensi

Cleusa Bianchi

Cleverson Busso

Clovis Antonio Rodrigues

Cornélio Alberto Zolin

Cristiane Kreutz

Cristiane Machado de López
University of Birmingham, Birmingham, Reino Unido

Escola Nacional de Saúde Pública Sergio Arouca (ENSP), Manguinhos, RJ, Brasil

University of Brescia, Brescia, Itália

Universidade Federal de Sergipe (UFS), São Cristóvão, SE, Brasil

Universidade de São Paulo (USP), São Paulo, SP, Brasil

Universidade de Franca (Unifran), Franca, SP, Brasil

Universidad Del Rosario, Bogotá, Colômbia

Instituto Federal de São Paulo (IFSP), Matão, SP, Brasil

Universidade do Estado do Rio de Janeiro (UERJ), Rio de Janeiro, RJ, Brasil

Universidade Federal do Rio Grande do Sul (UFRGS) Porto Alegre, RS, Brasil

Universidade Federal de Juiz de Fora (UFJF), Juiz de Fora, MG, Brasil

Universidade de Taubaté (UNITAU), Taubaté, SP, Brasil

Joint Research Centre (JRC), Ispra, Itália

Universidade Federal do Ceará (UFC), Fortaleza, CE, Brasil

Universidade do Vale do Itajaí, (UNIVALI), Itajaí, SC, Brasil

Cooperativa De Ensino Superior Politécnico Universitário (CESPU), Gandra, Portugal

Universidade Federal do Rio de Janeiro (UFRJ), Rio de Janeiro, RJ, Brasil

Universidade Federal de Minas Gerais (UFMG), Belo Horizonte, MG, Brasil

Universidade Federal do Ceará (UFC), Fortaleza, CE, Brasil

Universidade Estadual da Paraíba (UEPB), Campina Grande, PB, Brasil

Instituto de Aeronáutica e Espaço (IAE), São José dos Campos, SP, Brasil

Instituto Federal Catarinense (IFC), Blumenau, SC, Brasil

Universidade Regional do Noroeste do Estado do Rio Grande do Sul (Unijuí), Ijuí, RS, Brasil

Universidade Tecnológica Federal do Paraná (UTFPR), Dois Vizinhos, PR, Brasil

Universidade do Vale do Itajaí (UNIVALI), Itajaí, SC, Brasil

Embrapa Agrossilvipastoril, Sinop, MT, Brasil

Universidade Tecnológica Federal do Paraná (UTFPR), Campo Mourão, PR, Brasil

Pesquisa \& Desenvolvimento - Cemig (P\&D 486

Aneel/Cemig), MG, Brasil 


\section{Cristiano Christofaro}

Cynara de Lourdes da Nóbrega Cunha

Daiana Cardoso de Oliveira

Daniel Rodriguez

Daniele Maia Bila

Débora Cynamon Kligerman

Deborah Chapman

Deepak Jaiswal

Deise Ely

Dejanira de Franceschi de Angelis

Didem Okutman

Diogo Couto

Dominic Moran

Edna Regina Amante

Eduardo Beraldo Morais

Eduardo Cohim

Elaine Cristina Cardoso Fidalgo

Elenice Fritzsons

Eliane Pintor de Arruda

Elias Silva

Eline Simões Gonçalves

Elizabete Campos De Lima

Elton Camelo Marques

Elvira Carvajal

Evando Coelho

Evanilde Benedito
Universidade Federal dos Vales do Jequitinhonha e Mucuri (UFVJM), Teófilo Otoni, MG, Brasil

Universidade Federal do Paraná (UFPR), Curitiba, PR, Brasil

Universidade Federal de Santa Catarina (UFSC),

Florianópolis, SC, Brasil

Universidade Federal do Rio de Janeiro (UFRJ), Rio de Janeiro, RJ, Brasil

Universidade do Estado do Rio de Janeiro (UERJ), Rio de Janeiro, RJ, Brasil

Fundação Oswaldo Cruz (FIOCRUZ), Rio de Janeiro, RJ, Brasil

University College Cork (UCC), Cork, Irlanda

University of Illinois, Champaign, Illnois, Estados Unidos

Universidade Estadual de Londrina (UEL), Londrina, PR, Brasil

Universidade Estadual Paulista Julio de Mesquita Filho (UNESP), São Paulo, SP, Brasil

Istanbul Technical University, Istanbul, Turquía

Universidade de São Paulo (USP), São Paulo, SP, Brasil

Scotland's Rural College (SRUC), Edinburgh, Reino Unido

Universidade Federal de Santa Catarina (UFSC),

Florianópolis, SC, Brasil

Universidade Federal de Mato Grosso (UFMT), Cuiabá, MT, Brasil

Universidade Estadual de Feira de Santana (UEFS), Feira de Santana, Bahia, Brasil

Empresa Brasileira de Pesquisa Agropecuária (EMBRAPA), Rio de Janeiro, RJ, Brasil

Empresa Brasileira de Pesquisa Agropecuária (EMBRAPA), Brasília, DF, Brasil

Universidade Federal de São Carlos (UFSCar), Sorocaba, SP, Brasil

Universidade Federal de Viçosa (UFV), Viçosa, MG, Brasil

Fundação Oswaldo Cruz (FIOCRUZ), Rio de Janeiro, RJ, Brasil

Universidade Federal do ABC (UFABC), Santo André, SP, Brasil

Universidade Federal Rural do Semi-Árido (UFERSA), Mossoró, RN, Brasil

Universidade do Estado do Rio de Janeiro (UERJ), Rio de Janeiro, RJ, Brasil

Instituto Federal de Educação, Ciência e Tecnologia do Sul de Minas Gerais (IFSULDEMINAS), Pouso Alegre, MG, Brasil

Universidade Estadual de Maringá (UEM), Maringá, PR, Brasil 
Evelise Nunes Fragoso-Moura

Everlon Cid Rigobelo

Everton Skoronski

Evlyn Márcia Novo

Fábio Kummrow

Fábio Marcelo Breunig

Fabio Taioli

Fabrício Berton Zanchi

Felipe dos Santos Moysés

Fernando Cunha

Fernando Ferrari Putti

Fernando Gustavo Spaccesi

Fernando Lopez Vera

Fernando Mayer Pelicice

Fernando Ramos Martins

Figen Erkoc

Fillipe Tamiozzo Pereira Torres

Flávia Cristina Araújo Lucas

Flavia Mariani Barros

Francisco Carlos Lira Pessoa

Francisco de Assis de Oliveira

Francisco de Sousa Ramos

Francisco Holanda Nunes Junior

Francisco Silva Costa

Francisco Vanies da Silva Sá

Franklin Aragão Gondim

Gabriel Brito Costa
Universidade Federal de São Carlos (UFSCar), São Carlos, SP, Brasil

Universidade Estadual Paulista Júlio de Mesquita Filho (UNESP), Jaboticabal, SP, Brasil

Universidade do Estado de Santa Catarina (UDESC), Florianópolis, SC, Brasil

Instituto Nacional de Pesquisas Espaciais (INPE), São José dos Campos, SP, Brasil

Universidade Federal de São Paulo (UNIFESP), Diadema, SP, Brasil

Universidade Federal de Santa Maria (UFSM), Santa Maria, RS, Brasil

Universidade de São Paulo (USP), São Paulo, SP, Brasil

Universidade Federal do Sul da Bahia (UFSB), Eunápolis, BA, Brasil

Universidade Regional Integrada do Alto Uruguai e das Missões (URI), Erechim, RS, Brasil

Universidade Federal de Viçosa (UFV), Viçosa, MG, Brasil

Universidade Estadual Paulista Julio de Mesquita Filho (UNESP), Tupã, SP, Brasil

Centro Científico Tecnológico CONICET, La Plata, Argentina Universidad Autónoma de Madrid (UAM), Madrid, Espanha Universidade Federal do Tocantins (UFT), Palmas, TO, Brasil Universidade Federal de São Paulo (UNIFESP), Diadema, SP, Brasil

Gazi University, Ankara, Turquia

Universidade Federal de Viçosa (UFV), Viçosa, MG, Brasil

Universidade do Estado do Pará (UEPA), Belém, PA, Brasil

Universidade Estadual do Sudoeste da Bahia (UESB), Itapetinga, BA, Brasil

Universidade Federal do Para (UFPA), Belém, PA, Brasil

Universidade Federal Rural do Semi-Árido (UFERSA), Mossoró, RN, Brasil

Universidade Federal de Pernambuco (UFPE), Recife, PE, Brasil

Instituto Federal de Educação Ciência e Tecnologia do Ceará (IFCE), Jaguaribe, CE, Brasil

Universidade do Minho (UM), Braga, Portugal

Universidade Federal de Campina Grande (UFCG), Campina Grande, PB, Brasil

Instituto Federal de Educação, Ciência e Tecnologia do Ceará (IFCE), Maracanaú, CE, Brasil

Universidade Federal do Oeste do Pará (UFOPA), Belém, PA, Brasil 
Gabriel Constantino Blain

Gabriel Hornink

Gary Brierley

Gelze Rodrigues

George do Nascimento Ribeiro

George Kyzas

Geraldo César Rocha

German Andres Estrada Bonilla

Getulio Rincon Filho

Getulio Teixeira Batista

Gilberto Fernando Fisch

Gilmar Perbiche Neves

Gilson Barbosa Athayde Júnior

Giordano Urbini

Giovanni de Oliveira Garcia

Guilherme B. Lopes Jr

Guilherme Castioni

Guillaume Lentendu

Gundisalvo Piratoba Morales

Gustavo Cavalari Barboza

Gustavo Marques da Costa

Hanan Ghozlan

Han-Shen Chen

Hassan Fathizad

Hayet Djelal

Hélio Nobile Diniz

Heliofábio Barros Gomes

Heloisa Teixeira Firmo
Instituto Agronômico de Campinas (IAC), Brasil

Universidade Federal de Alfenas (UNIFAL), Alfenas, MG, Brasil

University of Auckland, Auckland, Nova Zelândia

Universidade Federal de Uberlândia (UFU), Uberlândia, MG, Brasil

Universidade Federal de Campina Grande (UFCG), Sumaré, PB, Brasil

Technological Educational Institute of Eastern Macedonia and Thrace, Kavala, Grécia

Universidade Federal de Juiz de Fora (UFJF), Juiz de Fora, MG, Brasil

Universidade de São Paulo (USP/ESALQ), Piracicaba, SP, Brasil

Universidade Federal do Maranhão (UFMA), Pinheiro, MA, Brasil

Universidade de Taubaté (UNITAU), Taubaté, SP, Brasil

Universidade de Taubaté (UNITAU), Taubaté, SP, Brasil

Universidade Federal de São Carlos (UFSCar), Buri, SP, Brasil

Universidade Federal da Paraíba (UFPB), João Pessoa, PB, Brasil

University of Insubria, Varese, Itália

Universidade Federal do Espírito Santo (UFES), Alegre, ES, Brasil

Universidade Federal do Triangulo Mineiro (UFTM), Uberaba, MG, Brasil

Universidade Estadual de Campinas (UNICAMP), Campinas, SP, Brasil

Technische Universität Kaiserslautern, Kaiserslautern, Alemanha

Universidade do Estado do Pará (UEPA), Belém, PA, Brasil

Universidade Estadual de Campinas (UNICAMP), Campinas, SP, Brasil

Universidade FEEVALE, Novo Hamburgo, RS, Brasil

Alexandria University, Alexandria, Egito

Chung-Shan Medical University Hospital Taichung, South District, Taichung, Taiwan

Yazd University, Yazd, Safaeih, Irã

Ecole Metiers L'environnement (EME), Bruz, França

Instituto Geológico do Estado de São Paulo (IG/SP), São Paulo, SP, Brasil

Universidade Federal de Alagoas (UFAL), Maceió, AL, Brasil Universidade Federal do Rio de Janeiro (UFRJ), Rio de Janeiro, RJ, Brasil 
Henrique Almeida

Herlander Mata-Lima

Herminia Yohko Kanamura

Holger Heuer

Hugo Abi Karam

Hugo Rafael Bentzen Santos

Ibraim Fantin-Cruz

Ifeanyi Francis Offor

Ignacio Dellaferrera

Ignacio Morell Evangelista

Ima Célia Guimarães Vieira

Iria Fernandes Vendrame

Isarita Martins

Ismael Maciel de Mancilha

Ivane Benedetti Tonial

Ivanildo Hespanhol

Jacqueline Bringhenti

Jairo Osvaldo Cazetta

James S. Latimer

Jane Marlei Boeira

Jaquelini Zeni

Jean Carlos Miranda

Jean Ricardo Simões Vitule

João Bassin

João Batitsta Lopes Silva

João Medeiros
Universidade Federal da Integração Latino-Americana

(UNILA), Foz do Iguaçu, PR, Brasil

Instituto Superior Técnico, Lisboa, Portugal

Universidade Federal de Alfenas (UNIFAL), Alfenas, MG, Brasil

Julius Kühn-Institut, Braunschweig, Alemanha

Universidade Federal do Rio de Janeiro (UFRJ), Rio de Janeiro, RJ, Brasil

Universidade Federal Rural de Pernambuco (UFRPE), Recife, PE, Brasil

Universidade Federal de Mato Grosso (UFMT), Cuiabá, MT, Brasil

Federal University Ndufu-Alike, Ikwo (FUNAI), Ikwo, Ebonyi, Nigeria

Universidad Nacional del Litoral, Esperanza, Argentina

University Jaume I- Pesticides and Water Research Institute, Espanha

Museu Paraense Emílio Goeldi, Belém, PA, Brasil

Instituto Tecnológico de Aeronáutica (ITA), São José dos Campos, SP, Brasil

Universidade Federal de Alfenas (UNIFAL), Alfenas, MG, Brasil

Escola de Engenharia de Lorena da Universidade de São Paulo, Lorena, SP, Brasil

Universidade Tecnológica Federal do Paraná (UTFPR), Francisco Beltrão, PR, Brasil

Universidade de São Paulo (USP), São Paulo, SP, Brasil

Instituto Federal de Educação Ciência e Tecnologia do Espírito Santo (IFES), Santa Teresa, ES, Brasil

Universidade Estadual Paulista Júlio de Mesquita Filho (UNESP), Jaboticabal, SP, Brasil

United States Environmental Protection Agency (EPA), Narragansett, Estados Unidos

Universidade Estadual do Rio Grande do Sul (UERGS), Novo Hamburgo, RS, Brasil

Universidade Estadual Paulista Julio de Mesquita Filho (UNESP), São José do Rio Preto, SP, Brasil

Universidade Federal Fluminense (UFF), Niteroi, RJ, Brasil

Universidade Federal do Paraná (UFPR), Curitiba, PR, Brasil

Universidade Federal do Rio de Janeiro (UFRJ), Rio de Janeiro, RJ, Brasil

Universidade Federal do Sul da Bahia (UFSB), Teixeira de Freitas, BA, Brasil

Marine and Environmental Sciences Centre (MARE), Lisboa, Portugal 
João Santos Nahum

João Sarkis Yunes

Joaquim Paulo da Silva

Joel Dias da Silva

John McCartney

Jonas Teixeira Nery

José Ángel González-Pérez

José Antonio Tosta dos Reis

José Carlos Mierzwa

José de Souza Nogueira

José Eloir Denardin

José Esteban Castro

José Luís Costa Novaes

José Luiz Negrão Mucci

José Luiz Silvério da Silva

José Maria Alves

José Ricardo Macedo Pezzopane

José Tavares Sousa

José Villegas_León

Julia Silva

Juliana Barreto Santos

Juliana Heloisa Pinê Américo-Pinheiro

Juliana Oliveira

Julio Cesar Pascale Palhares

Julio Cesar Raposo de Almeida

Jun-xia Liu
Universidade Federal do Pará (UFPA), Ananindeua, PA, Brasil

Universidade Federal do Rio Grande do Sul (UFRGS) Porto Alegre, RS, Brasil

Universidade Federal de Lavras (UFLA), Lavras, MG, Brasil

Universidade Regional de Blumenau (FURB), Blumenau, SC, Brasil

University of California San Diego, La Jolla, Estados Unidos

Universidade Estadual Paulista Júlio de Mesquita Filho (UNESP), Ourinhos, SP, Brasil

Universidad de Sevilla (US), Sevilla, Andalucía, Espanha

Universidade Federal do Espírito Santo (UFES), Vitória, ES, Brasil

Universidade de São Paulo (USP), São Paulo, SP, Brasil

Universidade Federal de Mato Grosso (UFMT), Cuiabá, MT, Brasil

Empresa Brasileira de Pesquisa Agropecuária (EMBRAPA), Passo Fundo, RS, Brasil

Newcastle University, Newcastle, Reino Unido

Universidade Federal Rural do Semi-Árido (UFERSA), Mossoró, RN, Brasil

Universidade de São Paulo (USP), São Paulo, SP, Brasil

Universidade Federal de Santa Maria (UFSM), Santa Maria, RS, Brasil

Universidade Estadual do Ceará (UECE), Fortaleza, CE, Brasil

Empresa Brasileira de Pesquisa Agropecuária (EMBRAPA), São Carlos, SP, Brasil

Universidade Estadual da Paraíba (UEPB), Campina Grande, PB, Brasil

Universidade Autônoma da Baja California (UABC), Mexicali, México

Universidade Federal de Minas Gerais (UFMG), Montes Claros, MG, Brasil

Universidade Federal do Rio de Janeiro (UFRJ), Rio de Janeiro, RJ, Brasil

Universidade Estadual Paulista Julio de Mesquita Filho (UNESP), Ilha Solteira, SP, Brasil

Centro de Pesquisas René Rachou (CPqRR), Belo Horizonte, MG, Brasil

Empresa Brasileira de Pesquisa Agropecuária (EMBRAPA), Embrapa Pecuária Sudeste, São Carlos, SP, Brasil

Universidade de Taubaté (UNITAU), Taubaté, SP, Brasil

State Key Lab Pollut Control, Shanghai, China 


\section{Katia Canil}

Keila Lima Sanches

Kelly Cristina Tonello

Kelly das Graças Fernandes Dantas

Kelly de Araujo Rodrigues Pessoa

Kenneth J. Boote

Klaas Metselaar

Lara Gabrielle Garcia

Laurel Schaider

Leandro Bugoni

Leandro Juen

Leila Limberger

Leo Fernandes Ávila

$\mathrm{Li} \mathrm{He}$

Lidia Lombardi Roma

Lidia Sas-Paszt

Liliana Pena Naval

Lincoln Eloi de Araújo

Lorenzo Marchi

Lucas Tadeu Fuess

Luciana Coelho Mendonça

Lucien Akabassi

Luciene Leite Rossi

Lucieta Martorano

Lucila Adriani Coral

Lucíola Lannes

Luís Antônio Coutrim Santos
Universidade Federal do ABC (UFABC), Santo André, SP, Brasil

Instituto Federal de Brasília (IFB), Brasília, DF, Brasil

Universidade Federal de São Carlos (UFSCar), Sorocaba, SP, Brasil

Universidade Federal do Pará (UFPA), Belém, PA, Brasil

Instituto Federal de Educação Ciência e Tecnologia do Ceará (IFCE), Fortaleza, CE, Brasil

University of Florida (UF), Gainesville, Flórida, Estados Unidos

Wageningen Universiteit (WUR), Wageningen, Países Baixos

Universidade de São Paulo (USP), São Paulo, SP, Brasil

Silent Spring Institute, Newton, Estados Unidos

Universidade Federal do Rio Grande do Sul (UFRGS) Porto Alegre, RS, Brasil

Universidade Federal do Para (UFPA), Belém, PA, Brasil

Universidade Estadual do Oeste do Paraná (UNIOESTE), Marechal Cândido Rondon, PR, Brasil

Universidade Federal de Pelotas (UFPel), Pelotas, RS, Brasil

North China Electric Power University, Beijing, China

Università degli Studi Niccolò Cusano (UNICUSANO), Roma, Itália

Instytut Ogrodnictwa, Skierniewice, Polônia

Universidade Federal do Tocantins (UFT), Palmas, TO, Brasil

Universidade Federal da Paraíba (UFPB), João Pessoa, PB, Brasil

Istituto di ricerca per la protezione idrogeologica (IRPI), Padova, Itália

Universidade de São Paulo (USP), São Carlos, SP, Brasil

Universidade Federal de Sergipe (UFSE), São Cristóvão, SE, Brasil

Instituto Federal de Educação, Ciência e Tecnologia do Espírito Santo (IFES), Vitória, ES, Brasil

Universidade Federal de São Carlos (UFSCar), Sorocaba, SP, Brasil

Empresa Brasileira de Pesquisa Agropecuária (EMBRAPA), Embrapa Amazônia Oriental, Belém, PA, Brasil

Universidade Tecnológica Federal do Paraná (UTFPR), Curitiba, PR, Brasil

Universidade Estadual Paulista Julio de Mesquita Filho (UNESP), Ilha Solteira, SP, Brasil

Universidade Federal de Santa Maria (UFSM), Santa Maria, RS, Brasil 
Luis Flávio Souza de Oliveira

Luiz Fernando Cappa de Oliveira

Luiz Guerreiro Lopes

Luiz Henrique Mazo

Luiz Olinto Monteggia

Lykke Andersen

M. Naushad

Magali Christe Cammarota

Manuela Santos-Pereira

Marcela Bianchessi Cunha-Santino

Marcelo Barcellos da Rosa

Marcelo Biudes

Marcelo de Paula Corrêa

Marcelo dos Santos Targa

Marcelo Gomes Silva

Marcelo Guerra Santos

Marcelo Henrique Otenio

Marcelo Shei

Marcia Matiko Kondo

Marcia Peiter

Marco Antonio Diodato

Marcus Emmanuel Mamana da Matta

Maria Anita Mendes

Maria de Lourdes Bueno Trindade Galo

Maria do Carmo Calijuri

Maria do Carmo Martins Sobral
Universidade Federal do Pampa (Unipampa), Bagé, RS, Brasil

Universidade Federal de Juiz de Fora (UFJF), Juiz de Fora, MG, Brasil

Universidade da Madeira (UMa), Ilha da Madeira, Funchal, Portugal

Universidade de São Paulo (USP), São Paulo, SP, Brasil

Universidade Federal do Rio Grande do Sul (UFRGS), Porto Alegre, RS, Brasil

Instituto de Estudios Avanzados en Desarrollo, La Paz, Bolívia

King Saud University, Riyadh, Arábia Saudita

Universidade Federal do Rio de Janeiro (UFRJ), Rio de Janeiro, RJ, Brasil

Universidade do Estado do Rio de Janeiro (UERJ), Rio de Janeiro, RJ, Brasil

Universidade Federal de São Carlos (UFSCar), São Carlos, SP, Brasil

Universidade Federal de Santa Maria (UFSM), Santa Maria, RS, Brasil

Universidade Federal de Mato Grosso (UFMT), Cuiabá, MT, Brasil

Universidade Federal de Itajubá (UNIFEI), Itajubá, MG, Brasil

Universidade de Taubaté (UNITAU), Taubaté, SP, Brasil

Instituto Nacional de Pesquisas Espaciais (INPE), São José dos Campos, SP, Brasil

Universidade do Estado do Rio de Janeiro (UERJ), Rio de Janeiro, RJ, Brasil

Empresa Brasileira de Pesquisa Agropecuária (EMBRAPA), Juiz de Fora, MG, Brasil

Altamar Equipamentos Aquáticos, Santos, SP, Brasil

Universidade Federal de Itajubá (UNIFEI), Itajubá, MG, Brasil

Universidade Federal de Santa Maria (UFSM), Santa Maria, RS, Brasil

Universidade Federal Rural do Semi-Árido (UFERSA), Mossoró, RN, Brasil

Ecoadvisor Associados, São Paulo, SP, Brasil

Universidade de São Paulo (USP), São Paulo, SP, Brasil

Universidade Estadual Paulista Julio de Mesquita Filho (UNESP), Presidente Prudente, SP, Brasil

Universidade de São Paulo (USP), São Paulo, SP, Brasil

Universidade Técnica de Berlin (TU BERLIN) Berlin, Alemanha 
Maria Eugênia Boscov

Maria Helena Rodrigues Gomes

Maria Isabel Sobral da Silva Escada

Maria Lucia Kolowski Rodrigues

Maria Lúcia Macedo Cardoso

Maria Lucrecia Gerosa Ramos

Maria Sueli Heberle Mafra

Mariana Heilbuth Jardim

Marianna Gilli

Marina Turini

Marinoé Gonzaga da Silva

Mario Takayuki Kato

Marney Pascoli Cereda

Martin Johannes Enk

Massimo Raboni

Maurício Alves da Motta Sobrinho

Maurício Capobianco Lopes

Mauricio Gustavo Coelho Emerenciano

Mauricio Perazzoli

Mauricio Pozzobon

Maurício Roberto Cherubin

Miguel Mansur Aisse

Milene Carvalho Bongiovani

Mirian Araujo Carlos Crapez

Mirian Fernanda Rodrigues

Mirtha Latsague

Mônica Luisa Kuhlmann
Universidade de São Paulo (USP), Butantã, SP, Brasil

Universidade Federal de Juiz de Fora (UFJF), Juiz de Fora, MG, Brasil

Instituto Nacional de Pesquisas Espaciais (INPE), São José dos Campos, SP, Brasil

Fundação Estadual de Proteção Ambiental Henrique Luis Roessler (FEPAM), Porto Alegre, RS, Brasil

Fundação Oswaldo Cruz (FIOCRUZ), Rio de Janeiro, RJ, Brasil

Universidade de Brasília (UnB), Brasília, DF, Brasil

Universidade do Planalto Catarinense (UNIPLAC), Lages, SC, Brasil

Faculdade Pitágoras (FPI), Guarapari, ES, Brasil

Universitá Degli Studi della Tuscia (UNITUS), Ferrara, Itália

Universidade Estadual Paulista Júlio de Mesquita Filho (UNESP), Rio Claro, SP, Brasil

Instituto Federal de Sergipe (IFS), São Cristóvão, SE, Brasil

Universidade Federal de Pernambuco (UFPE), Recife, PE, Brasil

Universidade Católica Don Bosco (UCDB), Campo Grande, MT, Brasil

Instituto Evandro Chagas (IEC), Ananindeua, PA, Brasil

LIUC - University "Cattaneo", School of Industrial

Engineering, Castellanza (VA), Itália

Universidade Federal de Pernambuco (UFPE), Recife, PE, Brasil

Universidade Regional de Blumenau (FURB), Blumenau, SC, Brasil

Universidade do Estado de Santa Catarina (UDESC), Florianópolis, SC, Brasil

Universidade do Oeste de Santa Catarina (UNOESC), Fraiburgo, SC, Brasil

Universidade Regional de Blumenau (FURB), Blumenau, SC, Brasil

Universidade de São Paulo (USP), Piracicaba, SP, Brasil

Universidade Federal do Paraná (UFPR), Curitiba, PR, Brasil

Universidade Federal de Mato Grosso (UFMT), Campo

Grande, MT, Brasil

Universidade Federal Fluminense (UFF), Niterói, RJ, Brasil

Universidade Federal de Santa Maria (UFSM), Santa Maria, RS, Brasil

Temuco Catholic University, Temuco, IX Región, Chile

Companhia Ambiental do Estado de São Paulo (CETESB), São Paulo, SP, Brasil 
Monise Abranches

Neeti Neeti

Nélia Henriques Callado

Nildo da Silva Dias

Noeli Júlia Schussler de Vasconcellos

Núbia Natália Brito

Omaira Omaira Márquez

Otidene Rossiter Sa da Rocha

Özfer Yesilada

Paolo Viotti

Patricia de Falco

Patrícia Franklin Mayrink Nogueira

Patrícia Pereira

Paul Withers

Paula Bacelar Nicolau

Paula Dias Bevilacqua

Paulina Maia-Barbosa

Paulo Cesar de Jesus

Paulo de Tarso Amorim Castro

Paulo Fortes Neto

Paulo Jorge de Campos Favas

Paulo Renato Matos Lopes

Paulo Rógenes Monteiro Pontes

Pedro Roberto Almeida Viégas

Philip Kerr

Pibief Goufo

Plínio Carlos Alvalá

Pooja Singh
Universidade Federal de Viçosa (UFV), Viçosa, MG, Brasil

TERI University, Delhi, Índia

Universidade Federal de Alagoas (UFAL), Maceió, AL, Brasil

Universidade Federal Rural do Semi-Árido (UFERSA), Mossoró, RN, Brasil

Centro Universitário Franciscano (UNIFRA), Santa Maria, RS, Brasil

Universidade Federal de Goiás (UFG), Goiânia, GO, Brasil

Universidad de Los Andes, El Vigía, Mérida, Venezuela

Universidade Federal de Pernambuco (UFPE), Recife, PE, Brasil

Inönü Üniversitesi, Battalgazi, Malatya, Turquia

Universita degli Studi di Roma La Sapienza, Roma, Itália

Universidade Federal de São Carlos (UFSCar), São Carlos, SP, Brasil

Universidade de São Paulo (USP), São Paulo, SP, Brasil

Universidade Federal do Rio Grande do Sul (UFRGS), Porto Alegre, RS, Brasil

Bangor University, Bangor, Reino Unido

Universidade Aberta (UAB), Lisboa, Portugal

Universidade Federal de Viçosa (UFV), Viçosa, MG, Brasil

Universidade Federal de Minas Gerais (UFMG), Belo Horizonte, MG, Brasil

Universidade Regional de Blumenau (FURB), Blumenau, SC, Brasil

Universidade Federal de Ouro Preto (UFOP), Ouro Preto, MG, Brasil

Universidade de Taubaté (UNITAU), Taubaté, SP, Brasil

Universidade de Trás-os-Montes e Alto Douro (UTAD), Vila Real, Portugal

Universidade Estadual Paulista Júlio de Mesquita Filho (UNESP), Dracena, SP, Brasil

Universidade Federal do Rio Grande do Sul (UFRGS) Porto Alegre, RS, Brasil

Universidade Federal de Sergipe (UFS), São Cristóvão, SE, Brasil

Charles Sturt University, Sydney, New South Wales, Austrália

Universidade de Trás-os-Montes e Alto Douro (UTAD), Vila Real, Portugal

Instituto Nacional de Pesquisas Espaciais (INPE), São José dos Campos, SP, Brasil

Symbiosis School of Biological Sciences (SSBS), Maharashtra, Índia 
Priscila Pereira Coltri

Rachel Bardy Prado

Rafael Albuquerque Xavier

Rafael Eduardo Chiodi

Raffaella Pomi

Raniere Barbosa de Lira

Raul Guimarães

Regina Teresa Rosim Monteiro

Renata Mendes de Carvalho

Renata Biagioni

Renata Ferraz Toledo

Renato Farias do Valle Junior

Renato Dantas Alencar

Renato Igor da silva Alves

Renato Molica

Ricardo Dagnino

Ricardo dos Santos Coelho

Ricardo Franci Gonçalves

Ricardo Mateus

Ricardo Santos Silva Amorim

Richard Ottermanns

Rodrigo Fernando dos Santos Salazar

Rodrigo Urban

Roger Babcock

Rogério Antonio Krupek

Rosane Barbosa Lopes Cavalcante
Universidade Estadual de Campinas (UNICAMP), Campinas, SP, Brasil

Empresa Brasileira de Pesquisa Agropecuária (EMBRAPA), Embrapa Solos, Rio de Janeiro, RJ, Brasil

Universidade Estadual da Paraíba (UEPB), Campina Grande, PB, Brasil

Universidade Federal de Lavras (UFLA), Lavras, MG, Brasil

Universita degli Studi di Roma La Sapienza, Roma, Itália

Cooperativa de Assessoria de Serviços (COOPERVIDA), Mossoró, RN, Brasil

Universidade Estadual Paulista Julio de Mesquita Filho (UNESP), Presidente Prudente, SP, Brasil

Universidade de São Paulo (USP), Piracicaba, SP, Brasil

Instituto Federal de Educação, Ciência e Tecnologia de Pernambuco (IFPE), Recife, PE, Brasil

Universidade Federal de São Carlos (UFSCar), Sorocaba, SP, Brasil

Universidade de São Paulo (USP), São Paulo, SP, Brasil

Instituto Federal de Educação, Ciência e Tecnologia do Triângulo Mineiro (IFTM), Uberaba, MG, Brasil

Instituto Federal do Rio Grande do Norte (IFRN), Mossoró, RN, Brasil

Universidade de São Paulo (USP), Ribeirão Preto, SP, Brasil

Universidade Federal Rural de Pernambuco (UFRPE),

Garanhuns, PE, Brasil

Universidade Estadual de Campinas (UNICAMP), Limeira, SP, Brasil

Instituto Federal de São Paulo (IFSP), São Roque, SP, Brasil

Universidade Federal do Espírito Santo (UFES), Vitória, ES, Brasil

Universidade do Minho (UM), Braga, Portugal

Universidade Federal de Mato Grosso (UFMT), Cuiabá, MT, Brasil

Rheinisch-Westfälische Technische Hochschule Aachen, Aachen, Nordrhein-Westfalen, Alemanha

Universidade de Cruz Alta (UNICRUZ), Cruz Alta, RS, Brasil

Universidade Estadual de Campinas (UNICAMP), Campinas, SP, Brasil

University of Hawaii, Hilo, Hawaii, Estados Unidos

Universidade Estadual do Paraná (UNESPAR), União da Vitória, PR, Brasil

Compañía Manufacturera de Papeles y Cartones/Celulose Riograndense (CMPC) Guaíba, RS, Brasil 
Roseli Lopes da Costa Bortoluzzi

Salvatore Grimaldi

Samuel Beskow

Sanjay Singh

Sarah Mello

Selma Regina Aranha Ribeiro

Sérgio Augusto Rodrigues

Sergio Byron Morera-Julca

Sergio Nascimento Duarte

Seyram Sossou

Shi-Peng Sun

Sibele Ezaki

Silvana de Paula Quintao Scalon

Silvia Helena Govoni Brondi

Silvia Helena Zanirato

Silvia Maria Ferreira Salvador

Silvia Vendruscolo Milesi

Silvio Bueno Pereira

Silvio Favero

Simon Costanzo

Simone Andrea Pozza

Simone Cardoso

Simone Maria Ribas Vendramel

Simone Rosa da Silva

Simoni Loverde-Oliveira

Sina Adl
Universidade do Estado de Santa Catarina - Campus III Planalto Serrano (UDESC), Lages, SC, Brasil

Universitá Degli Studi della Tuscia (UNITUS), Lazio, Itália

Universidade Federal de Pelotas (UFPel), Pelotas, RS, Brasil

Indian Agricultural Research Institute, New Delhi, Delhi, Índia

Universidade de São Paulo (USP), Piracicaba, SP, Brasil

Universidade Estadual de Ponta Grossa (UEPG), Ponta Grossa, PR, Brasil

Universidade Estadual Paulista Julio de Mesquita Filho (UNESP), Botucatu, SP, Brasil

Geophysical Institute of Peru (IGP), Cajamarca, Perú

Universidade de São Paulo (ESALQ-USP), Piracicaba, SP, Brasil

International Institute for Water and Environmental Engineering (2iE), Ouagadougou, Burkina Faso

Nanjing Tech University, Nanjing, Jiangsu, China

Instituto Geológico do Estado de São Paulo (IG/SP), São Paulo, SP, Brasil

Universidade Federal da Grande Dourados (UFGD), Dourados, MS, Brasil

Empresa Brasileira de Pesquisa Agropecuária (EMBRAPA), Embrapa Pecuária Sudeste, São Carlos, SP, Brasil

Universidade de São Paulo (USP), São Paulo, SP, Brasil

Universidade Estadual Paulista Júlio de Mesquita Filho (UNESP), Rio Claro, SP, Brasil

Universidade Regional Integrada do Alto Uruguai e das Missões (URI), Erechim, RS, Brasil

Universidade Federal da Grande Dourados (UFGD), Viçosa, MS, Brasil

Universidade Anhanguera (UNIDERP), Campo Grande, MS, Brasil

University of Maryland Center for Environmental Science, Cambridge, Estados Unidos

Universidade Estadual de Campinas (UNICAMP), Limeira, SP, Brasil

Universidade Federal de Juiz de Fora (UFJF), Juiz de Fora, MG, Brasil

Instituto Federal de Educação Ciência e Tecnologia do Rio de Janeiro (IFRJ), Rio de Janeiro, RJ, Brasil

Universidade de Pernambuco (UPE), Recife, PE, Brasil

Universidade Federal de Mato Grosso (UFMT),

Rondonópolis, MT, Brasil

College of Arts \& Science University of Saskatchewan, Saskatoon, Canadá 
Sonia Maria da Silva Carvalho

Sonja Germer

Stefan Stanko

Surender Reddy Salkuti

Susana Batel

Susana Rodriguez-Couto

Suymara Miranda

Suzana C. Wrublack

Suzete Gomes

Ta Yeong Wu

Taís Rondello Bonatti

Teresa Cristina Brazil de Paiva

Teresa Maria Reyna

Thiago Olitta Basso

Ticiana Marinho Carvalho Studart

Tomás de Aquino Portes e Castro

V. Kasuba

Vagner Roberto Elis

Valderi Duarte Leite

Valdir Eduardo Olivo

Vanessa Bezerra de Menezes Oliveira

Vanessa Corralo

Vanessa Retuci

Vera Lex Engel

Vera Vitali

Vicente Silva

Victor J García

Vidal Dias da Mota Júnior
Universidade Federal do Amazonas (UFAM), Manaus, AM, Brasil

Max Planck Institute for Biogeochemistry, Jena, Alemanha Slovak University of Technology in Bratislava, Faculty of Civil Engineering, Bratislava, Eslováquia

Indian Institute Of Technology Delhi, Delhi, Índia

Instituto Universitário de Lisboa (ISCTE), Lisboa, Portugal

Centro de Estudios e Investigaciones Técnicas de Gipuzkoa (CEIT), Donostia - San Sebastián, Espanha

Universidade Federal de Viçosa (UFV), Viçosa, MG, Brasil

Universidade Estadual do Oeste do Paraná (UNIOESTE), Cascavel, PR, Brasil

Fundação Oswaldo Cruz (FIOCRUZ), Rio de Janeiro, RJ, Brasil

Monash University, Subang Jaya, Selangor, Malásia

Universidade Paulista (UNIP), Campinas, SP, Brasil

Universidade de São Paulo (USP), Lorena, SP, Brasil

Universidad Nacional de Córdoba, Córdoba, Argentina

Universidade de São Paulo (POLI-USP), São Paulo, SP, Brasil

Universidade Federal do Ceará (UFC), Fortaleza, CE, Brasil

Universidade Federal de Goiás (UFG), Goiânia, GO, Brasil

University Zagreb, Zagreb, Croácia

Universidade de São Paulo (USP), São Paulo, SP, Brasil

Universidade Estadual da Paraíba (UEPB), Campina Grande, PB, Brasil

Universidade do Oeste de Santa Catarina (UNOESC), Chapecó, SC, Brasil

Universidade Estadual Paulista Julio de Mesquita Filho (UNESP), São Carlos, SP, Brasil

Universidade Comunitária Regional de Chapecó (UNOCHAPECÓ), Chapecó, SC, Brasil

Universidade Federal da Fronteira Sul (UFFS), Realeza, PR, Brasil

Universidade Estadual Paulista Julio de Mesquita Filho (UNESP), Botucatu, SP, Brasil

Instituto de Botânica (IBt), São Paulo, SP, Brasil

Universidade Federal de Campina Grande (UFCG), Campina Grande, PB, Brasil

Universidad de Los Andes, El Vigía, Mérida, Venezuela

Universidade de Sorocaba (UNISO), Sorocaba, SP, Brasil 
Vincenzo Torretta

Vinícius Batista Campos

Wallisson da Silva Freitas

Washington Franca-Rocha

Wei Lun Ang

Wellington Akira Iwamoto

Wouter Buytaert

Yuri Bezerra da Silva

Yushi Tian

Yvonilde Dantas Pinto Medeiros
Università degli Studi dell'Insubria, Varese, Itália

Instituto Federal de Educação, Ciência e Tecnologia do Amapá (IFAP), Laranjal do Jari, AP, Brasil

Instituto Federal de Educação Ciência e Tecnologia do Espírito Santo (IFES), Vitória, ES, Brasil

Universidade Estadual de Feira de Santana (UEFS), Feira de Santana, BA, Brasil

Universiti Kebangsaan Malaysia (UKM), Selangor, Malásia

Universidade Federal de Uberlândia (UFU), Uberlândia, MG, Brasil

Imperial College London, London, Reino Unido

Universidade Federal do Piaui (UFPI), Teresina, PI, Brasil

Harbin Institute of Technology (HIT), Haerbin Shi, Heilongjiang Sheng, China

Universidade Federal da Bahia (UFBA), Salvador, BA, Brasil

Note: 1) Some of these reviewers evaluated more than one manuscript;

2) This list includes reviewers that evaluated submissions that were not accepted for publication, in addition to the ones published in 2017.

As seen in Table 2, 90 reviewers are from international institution. This is an indication that the journal is gaining international acceptance and inclusion.

Table 2. Number and type of institutions of reviewers that contributed to Volume 12 of 2017.

\begin{tabular}{|c|c|c|c|c|c|c|}
\hline \multicolumn{5}{|c|}{ From Brazil } & \multicolumn{2}{|l|}{ From abroad } \\
\hline $\begin{array}{c}\text { Federal } \\
\text { Universities }\end{array}$ & $\begin{array}{c}\text { State } \\
\text { Universities }\end{array}$ & $\begin{array}{l}\text { State/Federal } \\
\text { Institutions }\end{array}$ & $\begin{array}{c}\text { National } \\
\text { private } \\
\text { Universities }\end{array}$ & $\begin{array}{c}\text { Municipal } \\
\text { Universities }\end{array}$ & $\begin{array}{c}\text { Reviewers from } \\
\text { international Institutions }\end{array}$ & Total \\
\hline 154 & 82 & 63 & 16 & 16 & 90 & 421 \\
\hline
\end{tabular}

